OU-TAP-70

December 1997

\title{
EVOLUTION OF THE POWER SPECTRUM AND THE SELF-SIMILARITY IN THE EXPANDING ONE-DIMENSIONAL UNIVERSE
}

\author{
Taihei Yano ${ }^{1}$ and Naoteru Gouda \\ Department of Earth and Space Science, Graduate School of Science, Osaka University \\ Toyonaka, Osaka 560, Japan \\ E-mail: yano, gouda@vega.ess.sci.osaka-u.ac.jp
}

\begin{abstract}
We have investigated time evolutions of power spectra of density fluctuations for long time after the first appearance of caustics in the expanding onedimensional universe. It is found that when an initial power spectrum is sale-free with a power index $n$, a self-similarity of the time evolution of the power spectrum is achieved. We find that the power spectrum can be separated roughly into three regimes according to the shape of the power spectrum: the linear regime $\left(k<k_{n l}\right.$ : the regime 1$)$, the single-caustic regime $\left(k_{n l}<k<k_{s n l}\right.$ : the regime 2$)$, and the multi-caustics regime $\left(k>k_{s n l}\right.$ : the regime 3$)$. The power index of the power spectrum in each regime has the values of $n,-1$, and $\mu$ which depends on $n$, respectively. Even in the case of an initial power-law spectrum with a cutoff scale, there might be the possibility of the self-similar evolution of the power spectrum after the appearance of the caustics. It is found, however, the self-similarity is not achieved in this case. The shape of the power spectrum on scales smaller than the cutoff scale can be separated roughly in two regimes: the virialized regime $\left(k_{c u t}<k<k_{c s}\right.$ : the regime 4$)$, and the smallest-single-caustic regime $\left(k>k_{c s}\right.$ : the regime 5$)$. The power index of the power spectrum is $\nu$ which may be determined by the distribution of singular points in the regime 4 . In the regime 5 , the value of the power index is -1 . Moreover we show the general property about the shape of a power spectrum with a general initial condition.
\end{abstract}

Subject headings: cosmology:theory-large scale structures-selfsimilar

\footnotetext{
${ }^{1}$ Research Fellow of the Japan Society for the Promotion of Science.
} 


\section{INTRODUCTION}

Formations of the large scale structures in the expanding universe is one of the most important and interesting problems of the cosmology. It is generally believed that these structures have been formed owing to gravitational instability. Hence it is very important to clarify evolutions of density fluctuations by the gravitational instability. Here we consider density fluctuations of collisionless particles as dark matters, our interest being mainly concentrated on effects of the self-gravity.

The density fluctuations are often characterized by their power spectrum (or two-point correlation function). Thus, it is very interesting and important to investigate time evolutions of the power spectrum. Furthermore, whether the evolutions of the power spectrum satisfy the self-similarity or not is especially important. If the self-similarity is satisfied, we can describe completely the time evolution of the power spectrum without numerical simulations because we can estimate the growth rate of the power spectrum on all scales. Many authors have investigated the self-similarity of the two-point correlation function (or the power spectrum). Davis \& Peebles (1977) investigated the self-similarity of the two-point correlation function by using the BBGKY equations. It is well known that we can make a similarity transformed BBGKY equations because the gravity is scale-free. They showed the existence of the self-similarity by integrating the BBGKY equations numerically under the assumptions as shown below. The BBGKY equations have the hierarchical structure, that is, the time evolution of the $N$-th order correlation function includes the $(N+1)$-th order correlation function. So, the cutoff of the hierarchy is needed in order to close these equations. Davis \& Peebles assumed that the three-point correlation function can be expressed by the product of the two-point correlation functions and that the skewness of the velocity field is equal to zero. Thus the existence of the self-similarity is still uncertain because we do not know whether these assumptions are correct or not. On the other hand, by using $N$-body simulations, time evolutions of the power spectrum can be calculated directly. Recent works, for example, are Colombi,Bouchet \& Hernquist (1996); Couchman \& Peebles (1997); Jain, Mo, \& White (1995) and so on. They showed that the self-similar evolution of the power spectrum can be satisfied when the initial power spectrum is scale-free. However the relation between the index of the power spectrum and the mean relative velocity is still uncertain quantitatively. Furthermore, only the scale-free cases are investigated. In this paper we would like to investigate not only the scale-free cases but also the cases that the initial power spectrum obeys the power-law with a cutoff. Hereafter we call this case cutoff-case. In the cutoff-case, the power spectrum obeys the power law even on scales smaller than the cutoff scale after the first appearance of caustics. For example, in the one-dimensional system, The value of the power index is -1 which can be derived according to the catastrophe theory (Gouda \& Nakamura $(1988,1989)$ ). Kotok 
\& Shandarin (1988) also studied the nonlinear spectra in the cutoff case and showed that the value of the power index is -1 . Of course, the power spectrum does not evolve in the self-similar form before the first appearance of caustics. However it has not been certain whether the time evolution is self-similar or not after the first appearance of caustics. Yano \& Gouda (1997) showed the possibility of the self-similarity in this case. Therefore we investigate the self-similarity not only in the case of the scale-free case but also in the cutoff-case. Then we have to investigate in detail the evolutions of the power spectrum on scales smaller than the initial cutoff scale and also those on larger scales. Thus we need a wide dynamic range in the wave number space. However, we cannot get such a wide dynamical range in the numerical simulation of three-dimensional systems due to the limit of the resolution. Then, as a first step, we consider one-dimensional sheet systems. In the one-dimensional system we can get a wide range of resolution for calculating time evolutions of the power spectrum. Furthermore we can have the numerical method for the evolution of the power spectrum with a good accuracy in the one-dimensional sheet systems as shown in $\S 2$. The purpose of this paper is to investigate the time evolution of the power spectrum in the one-dimensional systems as a first step. We are especially interested in the self-similar evolution of the power spectrum. Therefore, we consider only the background universe which does not have a characteristic scale, as a spatial curvature scale. That is, we consider the Einstein-de Sitter universe.

In $§ 2.1$, we will briefly show the numerical method. We show in $\S 2.2$ and $\S 2.3$ the numerical results of scale-free case, cutoff-case, respectively. Finally, we will devote to the conclusion and discussions in $\S 3$.

\section{NUMERICAL RESULTS}

\section{1. $\quad$ Numerical method}

We investigate time evolutions of the power spectrum and its self-similarity by using a numerical method. In this paper we consider the one-dimensional system that many plane parallel sheets move only a perpendicular direction to the surface of these sheets. When two sheets cross, they are allowed to pass through freely each other. In this sheet system, there is an exact solution until two sheets cross over as follows (Sunyaev \& Zel'dovich(1972), Doroshkevich et.al.(1973)):

$$
\begin{aligned}
& x=q+B_{1}(t) S_{1}(q)+B_{2}(t) S_{2}(q), \\
& v=\dot{B}_{1}(t) S_{1}(q)+\dot{B}_{2}(t) S_{2}(q),
\end{aligned}
$$


where $q$ and $x$ are the Lagrangian and the Eulerian coordinate, respectively. Here, $S_{1}(q)$, and $S_{2}(q)$ are arbitrary functions of $q . B_{1}(t)$, and $B_{2}(t)$ are the growing mode and the decaying mode of linear perturbation solutions, respectively. Since we consider the Einstein-de Sitter universe, $B_{1}(t)=a$ and $B_{2}(t)=a^{-\frac{3}{2}}$, where $a$ is a scale factor of the universe. We can compute the crossing time of all neighboring pairs of sheets. We use a shortest of these crossing time as a time step. Then, we can compute the new positions and velocities for all sheets at this crossing time. After two sheets cross, we exchange the velocities of just crossed two sheets. Then we obtain again $S_{1}(q), S_{2}(q)$, and therefore exact solutions as follows:

$$
\begin{gathered}
S_{1}(q)=\frac{3}{5} a^{-1}(x-q)+\frac{2}{5} \dot{a}^{-1} v, \\
S_{2}(q)=\frac{2}{5} a^{\frac{3}{2}}(x-q)-\frac{2}{5} \dot{a}^{-1} a^{\frac{5}{2}} v .
\end{gathered}
$$

These new exact solutions can be used until two sheets cross over. In this way we obtain the exact loci of the sheets by coupling these solutions. This is the numerical method with good accuracy because we connect the exact solutions. Through this paper, we use $2^{13}$ sheets for numerical calculation. A period boundary is fixed by a length of $2 \pi$. Therefore, the smallest wave number is 1 .

\subsection{Scale-free spectrum case}

In Figs.1(a) and (b), we show the time evolutions of the power spectrum with the scale-free initial power spectrum given by

$$
P\left(k, t_{i n i}\right) \propto k^{n},
$$

where $n$ is the power index of the initial spectrum. It is found that the following results are satisfied for $-1<n \leq 4$. The condition of $n>-1$ means the hierarchical clustering in the one-dimensional system. We are interested in this case $(n>-1)$ for "scale-free" case. One of the reasons why we consider this case is that the hierarchical clustering picture is expected in the real world. Another reason is that the results for $n<-1$ is similar to those in the case of the single wave case. See also eq.(14). Then the results for $n<-1$ can be reffered from the results shown in the regime 4 and 5 in the "cutoff case" (see §2.3). The condition of $n \leq 4$ is required because we consider the situation in which the non-linear mode coupling from higher $k$ to smaller $k$ can be neglected and so the linear perturbation theory can be hold on small $k$ ( Peebles (1980), Shandarin \& Melott (1990), Gouda (1995)). In the following, we show the case of $n=1$ and 2 for example. Fig.1(a) and(b) are the $n=1$ case and the $n=2$ case, respectively. We are interested in the hierarchical clustering 
picture. Therefore we chose some examples that have the power index larger than -1 . We can obtain the same results qualitatively in the cases of the other indexes. Here $t_{i n i}$ is the initial time, and the initial scale factor of these two cases are 0.1 and 0.01 , respectively. We normalize the scale factor as follows; $a=1$ when the first caustic has appeared. The phases of the initial fourier spectrum are given in random. We have averaged 50 samples. Here, we scale the wave number and power spectrum as follows:

$$
k_{*} \equiv \frac{k}{k_{n l}(t)}, \quad P_{*}\left(k_{*}, t\right) \equiv \frac{P(k, t)}{P_{\text {scale }}(t)},
$$

where $k_{n l}$ is defined by

$$
\frac{1}{2 \pi} \int_{0}^{k_{n l}(t)} P(k, t) d k=1
$$

In the regime of $k<k_{n l}$, the power spectrum grows according to the solution of linear perturbation, that is, the power spectrum satisfies the relation $P(k, t) \propto a^{2}$. Therefore, $k_{n l}$ is proportional to $a^{-2 /(n+1)}$. $P_{\text {scale }}(t)$ is defined by

$$
P_{\text {scale }}(t) \equiv P\left(k_{n l}(t), t\right)
$$

Then $P_{\text {scale }}(t)$ is proportional to $a^{2} k_{n l}^{n} \propto a^{2 /(n+1)}$. This scaled power spectrum $P_{*}\left(k_{*}\right)$ is shown in Fig.2(a) for the case of $n=1$. The same one but for the case of $n=2$ is shown in Fig.2(b). We can see the coincidence of each power spectrum at each time with good accuracy. This means that the self-similarity is achieved in the scale-free case. Furthermore we can see the three different power indexes in three regimes: the linear regime ( $k<k_{n l}$ : the regime 1$)$, the single-caustic regime $\left(k_{n l}<k<k_{s n l}\right.$ : the regime 2$)$, and the multi-caustics regime $\left(k>k_{\text {snl }}\right.$ : the regime 3$)$. Fig.3 shows the schematic general power spectrum at a time in the scale-free case. The value of the power index in the linear regime, of course, remains unchanged. We explain the power index of the power spectrum in the other two regimes: the single-caustic regime, and the multi-caustics regime in the following subsections.

\subsubsection{Single-caustic regime (the regime 2)}

In the single-caustic regime $\left(k_{n l}<k<k_{s n l}\right.$ : the regime 2$)$ we can see that the power index value becomes -1 . The reason is as follows; The density perturbation with the scale which just entered the non-linear regime at a given time, that is, $k_{n l}$, makes "caustics". Strictly speaking, the caustics would be appeared if the initial spectrum was smoothed bellow the scales just entered the non-linear regime. Therefore, we must notice that the real caustics cannot be observed in the scale-free case because of smearing by the small scale 
fluctuations. However we call these scale on $k_{n l}<k<k_{\text {snl }}$, "single-caustics regime" in this paper. After the first appearance of "caustics", the value of the power index of the power spectrum on the scales smaller than that scale is -1 (Gouda \& Nakamura (1989), Kotok \& Shandarin (1988)): Here we briefly show why the value of the power index is -1 after the first appearance of caustics.

The fourier spectrum $\delta_{k}$ of the density fluctuation is given by

$$
\delta_{k}=\int \delta(x) e^{i k x} d x
$$

Then the density is given by

$$
\rho(x)=\frac{\rho_{0}}{\left|\frac{d x}{d q}\right|},
$$

where $\rho_{0}$ is the mean density of the universe. At the Lagrangian singular point $q_{0}$, the following relation is satisfied.

$$
\left(\frac{d x}{d q}\right)_{q_{0}}=0
$$

At the singular point $q_{0}$, the density $\rho(x)$ diverges. Therefore, the Eulerian coordinate $x$ can be written

$$
x=x_{0}+\beta\left(q-q_{0}\right)^{2}+O\left(\left(q-q_{0}\right)^{3}\right) .
$$

For simplicity, we can put $x_{0}=q_{0}=0$ without losing generality. Therefore we can express $x=\beta q^{2}$ around the caustic. The density around the caustic is expressed by

$$
\rho(x)=\rho_{0}\left|\frac{d x}{d q}\right|^{-1} \propto(\beta x)^{-\frac{1}{2}}
$$

This density profile determine the proper index of the power spectrum. This type of singularity is called A2 type according to the catastrophe theory (Gouda \& Nakamura $(1988,1989))$. Eqs.(10), and (11) can be generally satisfied around the singular points in the multi-stream flow regimes where the Zel'dovich solution cannot hold. The A2 type of singularity is the only stable type in the one-dimensional system and their eqs.(10), and (11) remain in the multi-stream flow regions after the first appearance of caustics. Here it must be noted that Lagrange coordinate $q$ in the above argument is not the initial position of the sheet. Please refer to Roytvarf (1987), and Gouda \& Nakamura (1989), for the detailed explanation. On the other hand, it is found that $\rho(x)$ is proportional to $x^{-2 / 3}$ around the singular points at the first appearance of caustics (Zel'dovich (1970), Arnold et.al.(1982), Gouda \& Nakamura (1988)). This type of singularity is called A3 type. The A3 Type is not structural stable in the one-dimensional system and then the A3 type of singularities appears only at an instant. It disappears and quickly evolve into the A2 type, i.e. $\rho \propto x^{-1 / 2}$ 
singularity. Hence the contribution from the A3 type singularity in estimating the power spectrum is negligible. The fourier spectrum of the density is given by

$$
\begin{aligned}
\delta_{k} & =\int \delta(x) e^{i k x} d x \\
& =\int \frac{\rho}{\rho_{0}} e^{i k x} d x \\
& \propto \int(\beta x)^{-\frac{1}{2}} e^{i k x} d x \\
& =\beta^{-\frac{1}{2}} k^{\frac{1}{2}} k^{-1} \int t^{-\frac{1}{2}} e^{i t} d t,
\end{aligned}
$$

where $t \equiv k x$. Then, we obtain

$$
P(k) \propto(k \beta)^{-1} .
$$

From eq.(13), we find the value of the power index is -1 . Here we notice that small $\beta$ contributes to the large amplitude of the power spectrum. Furthermore, the coefficient $\beta$ is very small when the caustics have just appeared. We can see this fact by the evolution of the "single-wave perturbation". Here, the single-wave perturbation means the density fluctuation whose initial condition is given by

$$
x(q)=q+X \sin (q),
$$

where $X$ is a constant value. Hereafter we call this case the single-wave case. The first caustics have just appeared at a center of the $x$ axis $(x=0)$ in Fig.4(a). After the first appearance of this caustic, more caustics appear in the collapse regime by phase mixing (Fig.4(b)-(d)) (Doroshkevich et.al(1980), Melott (1983), Gouda \& Nakamura (1989)). A caustic (singularity of the density field) is located at the point where the derivative of $v$ with respect to $x$ is infinity. The absolute value of the derivative of $v$ with respect $x$ around the singular points is proportional to $\beta^{-1}$. Then as the phase mixing continues and so more caustics appear, it is found from Fig.4(a)-4(d) that $\beta$ increase because $\left|\frac{d v}{d x}\right|$ around the singular points decrease. Hereafter we call the structure in the phase space shown in Fig.4 (a)-(b) "the whirlpool". Just after the multi-caustics have appeared, the coefficient factor $\beta$ of this wavelength is very small and the effect of the amplitude of the power spectrum in these waves dominates. As a result, the index of -1 that is predicted by the catastrophe theory appears. Therefore we call these regimes, single-caustic regime.

Here, we comment that in the real singularities do not occur in the real world. Of course, the arguments on the caustics in this paper cannot be applied to barionic gas because shocks appeared and they prohibited that the density diverges. Furthermore the maximum density is surprisingly low even in the neutrino-dominated model due to their velocity dispersion (Zel'dovich \& Shandarin (1982),Kotok \& Shandarin (1987)). In this paper, we consider the cold collisionless matters whose velocity dispersion is much small compared with the scales under consideration. 


\subsubsection{Multi-caustics regime (the regime 3)}

In the multi-caustics regime $\left(k>k_{s n l}\right)$, we can see the power law spectrum. With the power index of these regimes is different from -1 predicted by catastrophe theory. In this regime, various small scale fluctuations $\left(k \gg k_{s n l}\right)$ have already collapsed and made singularities. Every singularity makes "the whirlpool" in phase space as shown in the single-wave case (Fig.4). Various size of "whirlpools" are made of the various scale of fluctuations. The distribution of the whirlpools determine the value of the power index. This distribution depends on the initial power index $n$. That is, the power index $\mu$ in this regime depends on $n$. Indeed, the value $\mu$ depends on the initial power spectrum as shown in Fig.2(a), and 2(b). Therefore we call this regime the multi-caustics regime. When the self-similarity is satisfied, there is a relation between the power index of the two-point correlation function, the initial power spectrum index, and the mean relative peculiar velocity. Here, we briefly show this relation in the $w$ dimensional system. In the linear regime of the $w$ dimensional system, the two-point correlation function is given by(Peebles 1980,1993),

$$
\xi \propto a^{2} x^{-(w+n)} \propto\left(\frac{x}{a^{\alpha}}\right)^{-(w+n)}
$$

where

$$
\alpha \equiv \frac{2}{w+n} .
$$

On the other hand, in the strongly nonlinear regime, the two-point correlation function obeys the following evolution equation (0th moment equation of the 2nd BBGKY eq.: Davis \& Peebles (1977), Yano \& Gouda (1997)),

$$
\frac{\partial \xi}{\partial t}+\frac{1}{a x^{(w-1)}} \frac{\partial}{\partial x}\left[x^{(w-1)}(\xi+1)\langle v\rangle\right]=0 .
$$

In the strongly non-linear limit, we can assume a power law solution for the two-point correlation function (Yano \& Gouda (1997)).

$$
\xi \propto a^{\epsilon} x^{-\gamma}
$$

In this case, we obtain

$$
\langle v\rangle=-h \dot{a} x, \quad(\epsilon-h(w-\gamma)=0)
$$

and then,

$$
\xi \propto a^{h(w-\gamma)} x^{-\gamma}=\left(\frac{x}{a^{\alpha^{\prime}}}\right)^{-\gamma},
$$


where $h$ is the constant and

$$
\alpha^{\prime} \equiv \frac{h(w-\gamma)}{\gamma} .
$$

Hereafter we call the parameter $h$ the relative velocity parameter. If the self-similarity is satisfied, $\alpha^{\prime}$ must be equal to $\alpha$. Then the power index $\gamma$ is given by

$$
\gamma=\frac{w h(n+w)}{2+h(n+w)} .
$$

If the relative velocity parameter $h$ is 1 (stability condition) as Davis \& Peebles (1977) assumed, we obtain the $\gamma=(n+1) /(3+n)$ in the one-dimension $(w=1)$. In this case, the power index $\mu$ of the power spectrum is given by $\mu=\gamma-w=-2 /(3+n)$. However the power index $\mu$ which is given by the numerical results of the power spectrum is different from $-2 /(3+n)$. Therefore, the stability condition $(h=1)$ is not satisfied. We can estimate the velocity parameter $h$ from the power index by using eq.(22).

$$
h=\frac{2 \gamma}{(n+w)(w-\gamma)}=-\frac{2(\mu+w)}{(n+w) \mu}=-\frac{2(\mu+1)}{(n+1) \mu} .
$$

We show the results in the Table 1. Yano \& Gouda (1997) discussed that the velocity parameter $h$ has the value between 0 and 1 . Indeed the value of $h$ stays at this range. We show the numerical result of $h$ not only for the $n=1$, and 2 cases but also for the $n=0$, and 3 cases.

Table 1. $n$ dependence of $\mu$ and $h$

\begin{tabular}{ccc}
$n$ & $\mu$ & $h$ \\
\hline 0 & -0.88 & 0.29 \\
1 & -0.75 & 0.33 \\
2 & -0.65 & 0.36 \\
3 & -0.58 & 0.37
\end{tabular}

\subsection{Cutoff-case}

We consider the following initial spectrum, that is, the cutoff-case.

$$
P(k)\left\{\begin{array}{cc}
\propto k & \left(k<k_{\text {cut }}\right) \\
=0 & \left(k>k_{\text {cut }}\right)
\end{array} .\right.
$$

The time evolution of the power spectrum for the cutoff-case is shown in Fig.5. It is shown only for the $n=1$ case. However, we obtain essentially the same result for other cases 
( $n=2,3$, and 0 ). As we can see from Fig.5, until the first appearance of caustics (lowest solid line of the power spectrum except for initial spectrum in Fig.5), the amplitude of the power spectrum grows even on scales smaller than the cutoff scale. The power index of these regime is predicted to be -1 . Because the wave number $k=k_{\text {cut }}$ makes the caustic and this caustic results in the predicted value of power index as shown in $\S 2.2$. Indeed, we find that the value of the index in these regimes is nearly equal to -1 . The scaled power spectrum is also shown in Fig.6. The definition of the scaling is the same as that in the scale-free case. As we see from Fig.6, each power spectrum at the different time does not coincide. Therefore the self-similarity is not satisfied.

The characteristic scale of separation of the caustics becomes smaller and smaller as time increases (refer to the case of the single-wave: See Fig.4 (a)-(d)). Here, $k_{c s}$ is defined as the wave number of the characteristic scale. On the scales smaller than the characteristic scale $k>k_{c s}$ (regime 5: See Fig.10), the power index of the power spectrum becomes -1 . Because on the scales smaller than the characteristic scale, the density profile around one singular point determines the power spectrum. Therefore we call this regime the smallest single-caustic regime. On the other hand, the regime $k_{c u t}<k<k_{c s}$ (regime 4 ), the power index of the power spectrum is different from -1 . The index has a certain value $\nu$. Because on the scales larger than the characteristic scale, smoothed density profile with the smoothing scale $\left(k_{c u t}<k<k_{c s}\right)$ determine the power spectrum. We call this regime the virialized regime. We show the schematic of the density distribution in Fig.7. We notice that the wave number $k_{c s}$ which represents the characteristic separation of caustics becomes larger and larger as time increases. On the contrary, the non-linear scale wave number $k_{n l}$ and $k_{s n l}$ become smaller and smaller as time increases. Thus, the power spectra of the different times cannot coincide on all scales. Therefore the self-similar evolution in the all regimes including the scales smaller than the cutoff scale cannot be satisfied.

We compare the time evolutions of the power spectrum for the cutoff-case with those for the scale-free case in Fig.8. Of course, the power spectrum for these two cases does not coincide with each other on all scales. On the other hand, on the scales larger than the cutoff scale, we can see the coincidence of power spectra for these two cases. This means that the growth rate on the wave number smaller than $k_{c u t}$ does not affect the difference of the larger wave number than the cutoff scale. We show the scaled power spectrum for the cutoff-case only on the wave numbers smaller than the cutoff scale $k_{\text {cut }}$ in Fig.9. This means that even in the cutoff-case, the self-similar evolution can be satisfied on scales larger than the cutoff scale. 


\section{CONCLUSIONS AND DISCUSSION}

We have calculated the time evolution of the power spectrum for two cases of initial conditions. One is the scale-free case and the other one is the cutoff-case. In the case of the scale-free case, we can see the self-similar evolution of the power spectrum. The scaled power spectrum $P_{*}\left(k_{*}\right)$ at each time coincides with each other. We can separate roughly the power spectrum into three regimes. One is the linear regime $\left(k<k_{n l}\right.$ : the regime 1$)$. The value of the power index in this regime remains the initial power index, $n$. The second regime is the single-caustic regime $\left(k_{n l}<k<k_{s n l}\right.$ : the regime 2$)$. In this regime, the power index becomes -1 and is independent of the initial conditions. This result is caused by the appearance of caustics at this scale, and these caustics determine the power index of the power spectrum in this regime. The third regime is the multi-caustics regime $\left(k>k_{s n l}\right.$ : the regime 3). The distribution of the "whirlpool" in phase space determine the value of the power index. Therefore in this scale the power index $\mu$ has the value which depends on the initial condition. We can estimate the velocity parameter $h$ from the power index in the multi-caustics regime, which is around 0.5. Yano \& Gouda (1997) discussed the probable value of $h$ from the physical point of view, and obtained that $h$ takes a value between 0 and 1. Estimated values of $h$ are consistent with this argument. Indeed, the index $\mu$ and the velocity parameter $h$ depend on the initial power index $n$. The stability condition $(h=1)$ is not satisfied in this case.

In the cutoff-case, we find that there is no self-similarity on all scales. The scaled power spectrum does not coincide with each other in all regimes. However, the spectrum coincides on the scales larger than the cutoff scale. After the appearance of the first caustics, the power index in the regime of the scale smaller than the cutoff scale becomes -1 . This value is, as mentioned above, caused by the appearance of caustics as shown in Fig.3. More and more caustics appear one after another and so the separation of caustics becomes smaller and smaller. Even after the appearance of many caustics, on scales smaller than the characteristic separation of the caustics $\left(k>k_{c s}\right.$ : the regime 5$)$, the power index of the power spectrum is obtained by the density profile around the singular point. Therefore the power index of these scales becomes -1 which can be derived according to the catastrophe theory. On the other hand, on the scales larger than the characteristic separation of the caustics $\left(k_{c u t}<k<k_{c s}\right.$ : the regime 4$)$, the distribution of the singular points determines the power index on these scales instead of the density profile around one singularity. Because in this regime, the smoothed density profile with the smoothing scale $\left(k_{c u t}<k<k_{c s}\right)$ determine the power index. On these scales, the distribution of the singularity occurring in the evolution of the single-wave is important. Then, the power index on these scales is determined by this distribution of the singularity. Therefore, we can roughly separate two regimes on the scales smaller than the cutoff scale $k_{c u t}$. One is 
the virialized regime $\left(k_{c u t}<k<k_{c s}\right.$ : the regime 4), and the power index has the value $\nu$. Another is the smallest single-caustic regime $\left(k>k_{c s}\right.$ : the regime 5$)$ and the index have the value of -1 . Both indexes are independent of the initial conditions.

In a real situation about the evolution of the power spectrum, the initial power spectrum has a cutoff at a certain scale. Therefore, in the case of a real situation, we can consider the evolution of the cutoff-case. We notice that even if the initial power spectrum does not have a cutoff, it decreases with the power index $n<-1$ on certain scales $\left(k>k_{d e c}\right)$. Therefore the time evolution of the power spectrum is the same with the one in the cutoff-case after the appearance of caustics. And then, we can separate roughly five regimes. We show a schematic of the power spectrum at a time after the first appearance of caustics in Fig.10. First regime is the linear regime $\left(k<k_{n l}\right.$ : the regime 1). The index of the power spectrum in this regime is $n$. Second regime is the single-caustic regime $\left(k_{n l}<k_{s n l}\right.$ : the regime 2). The index in this regime is -1 , and independent of the initial conditions. Third regime is the multi-caustics regime $\left(k_{\text {snl }}<k_{\text {cut }}\right.$ : the regime 3$)$. The power index is $\mu$ which depends on the initial power index $n$. Fourth regime is virialized regime $\left(k_{c u t}<k_{c s}\right.$ : the regime 4$)$. The value of the power index is $\nu$ which is independent of the initial power index. The fifth regime is the smallest single-caustic regime $\left(k>k_{c s}\right.$ : the regime 5). The value of the power index is -1 . Only when we consider the evolution of the power spectrum on scales larger than the cutoff scale (i.e. the first, second, and third regime), the self-similarity is satisfied.

We would like to thank T.Tanaka for important comments. And we would like to thank E. Van Drom for useful suggestions. We are grateful to the referee, S.F. Shandarin for many useful and important suggestions. This work was supported in part by Research Fellowships of the Japan Society for the Promotion of Science for Young Scientists (No.4746).

\section{REFERENCES}

Arnol'd, V.I., Shandarin, S.F. \& Zel'dovich, Ya.B. 1982, Geophys. Astrophys. Fluid Dynamics 20, 111

Colombi, S., Bouchet, F.R. \& Hernquist,L. 1996, ApJ, 465, 14

Couchman, H.M.P. \& Peebles, P.J.E. 1997, preprint astro-ph/9708230

Davis, M., \& Peebles, P.J.E. 1977, ApJS, 34, 425 (DP)

Doroshkevich, A.G., Ryaben'kii, V.S. \& Shandarin, S.F. 1973, Astrophysics, 9, 144 
Doroshkevich, A.G., Kotok, E.V., Novikov, I.D., Polyudov, A.N., Shandarin, S.F.\& Sigov, Yu.S. 1980, MNRAS, 192, 321

Fillmore, J.A., \& Goldreich, P. 1984, ApJ, 281, 9

Gouda, N. 1995, Prog.Theor.Phys., 94, 33

Gouda, N. \& Nakamura, T. 1988, Prog.Theor.Phys., 79, 765

Gouda, N. \& Nakamura, T. 1989, Prog.Theor.Phys., 81, 633

Gurevich, A.V. \& Zybin, K.P. 1995 Physics-Uspekhi, 38, 687

Jain, B.,Mo, H.J. \& White, S.D.M., 1995, MNRAS, 276, L25

Kotok, E.V. \& Shandarin, S.F. 1987, Soviet Astr., 31, 600

Kotok, E.V. \& Shandarin, S.F. 1988, Soviet Astr., 32, 351

Melott, A.L. 1983, ApJ, 264, 59

Peebles, P.J.E. 1980, The Large-Scale Structure of the Universe (Princeton:Princeton Univ.Press)

Peebles, P.J.E. 1993, The Principles of Physical Cosmology (Princeton:Princeton Univ.Press)

Roytvarf, A. 1987, Vestnik. Moscow University. Ser.1, No.1, 65

Roytvarf, A. 1987, Vestnik. Moscow University. Ser.1, No.3, 41

Shandarin, S.F. \& Melott, A.L. 1990, ApJ364, 396

Sunyaev, R.A. \& Zel'dovich, Ya.B. 1972, A\&A, 20,189

Yano, T. \& Gouda, N. 1997, ApJ, 487, 473

Zel'dovich, Ya.B. 1970, A\&A, 5, 84

Zel'dovich, Ya.B. \& Shandarin, S.F. 1982 Sov. Astron. Lett., 8, 139 
Fig.1.-(a) The time evolution of the power spectrum. The solid and dotted lines are used mutually in order to distinguish easily each line. The curves shown are (bottom to top) at $a=0.1,1,2,4,8,16,32,64,128$. The initial power spectrum is scale-free with the power index $n=1$ (Dotted-dashed line).

(b) The same as (a) but for the initial power index $n=2$ and the power spectra are shown at the scale factor $a=0.01,1,2,4,8,16,32,64,128$.

Fig.2.-(a) The scaled power spectrum $P_{*}\left(k_{*}\right)$. The initial power spectrum is scale-free with the power index $n=1$. Three solid straight line shows the power law with the power index of $1,-1,-0.75$.

(b) The same as (a) but for the initial power index $n=2$. Three solid straight line shows the power law with the power index of $2,-1,-0.65$.

Fig.3.- The schematic of the power spectrum in the scale-free case at a certain time.

Fig.4.-(a) The distribution in the phase space for the single-wave case at the first appearance of caustics. A horizontal axis and a vertical axis are the Eulerian coordinate $x$ and the velocity, respectively. Scale factor $a$ is normalized at this time $(a=1)$.

(b) The same as (a) but at $a=4$.

(c) The same as (a) but at $a=16$.

(d) The same as (a) but at $a=32$.

Fig.5.- The time evolution of the power spectrum. The solid and dotted lines are used mutually in order to distinguish easily each line. The curves shown are (bottom to top) at $a=0.05,1,2,4,8,16,32,64,128$. The initial power spectrum obeys the power-law with the index $n=1$ and it has a cutoff at $k_{c u t}=127$ (Dotted-dashed line).

Fig.6.- The scaled power spectrum $P_{*}\left(k_{*}\right)$. The initial power spectrum obeys the power-law with the index $n=1$ and it has a cutoff scale at $k_{\text {cut }}=127$.

Fig.7.- The schematic of the density distribution in the single-wave case. This figure shows that the density profile around one caustic and the distribution of caustics, which determine the smoothed density profile on scales larger than the characteristic separation of caustics.

Fig.8.- Comparison of the evolution of the power spectrum between the scale-free case (solid line) and the cutoff-case (dashed line). Both the solid curves and dashed curves shown are (bottom to top) at $a=0.05,1,2,4,8,16,32,64$. The initial power spectrum obeys the power law with the power index $n=1$ both for the scale-free case and for the cutoff-case. For the cutoff-case, the initial power spectrum has a cutoff at $k_{\text {cut }}=127$. 
Fig.9.- The scaled power spectrum at $\mathrm{a}=1,2,4,8,16,32,64$ on scales larger than the cutoff scale. The initial power spectrum obeys the power law with the power index $n=1$ and it has a cutoff scale at $k_{\text {cut }}=127$.

Fig.10.- The schematic of the power spectrum with a general initial power spectrum at a certain time after the first appearance of caustics. 


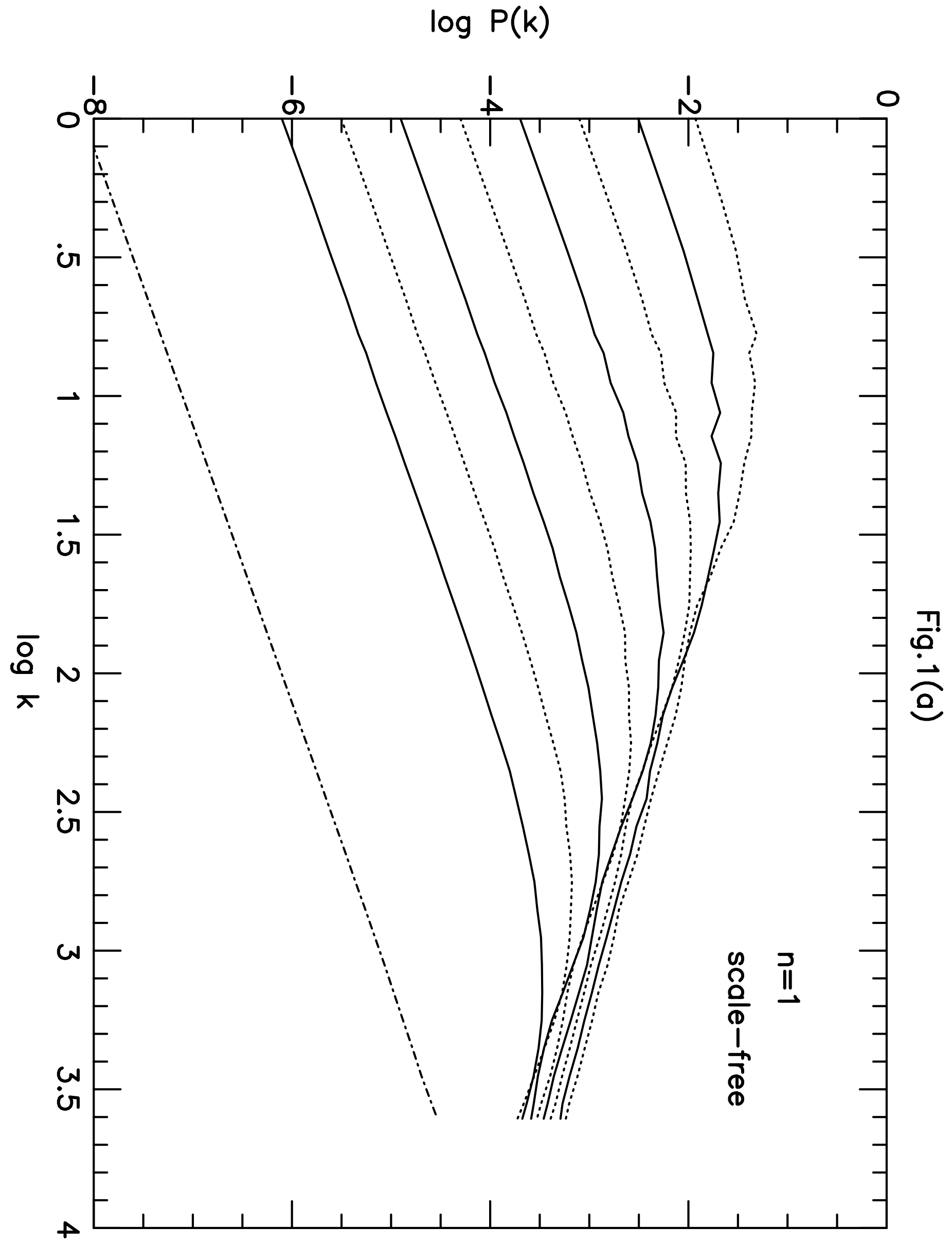




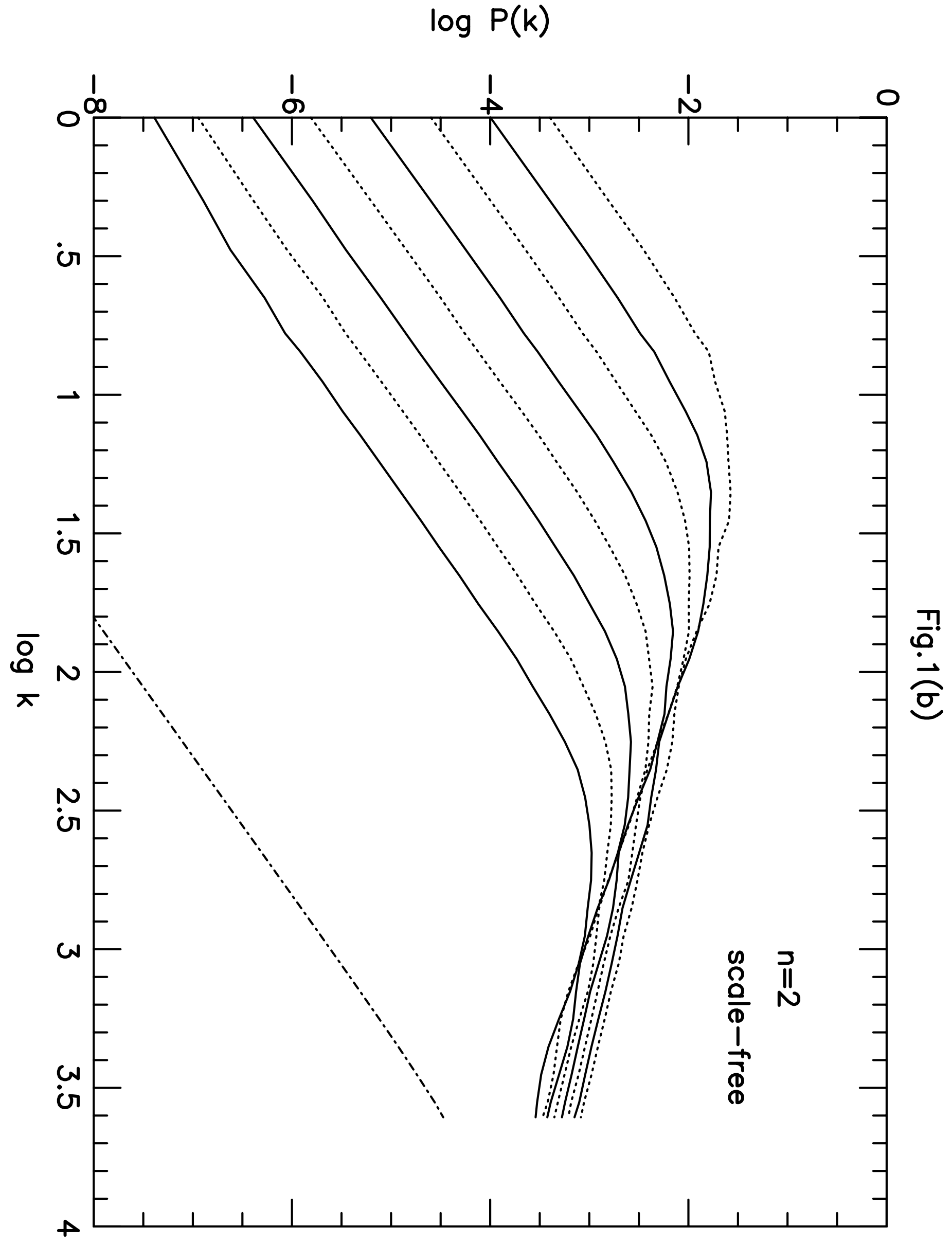




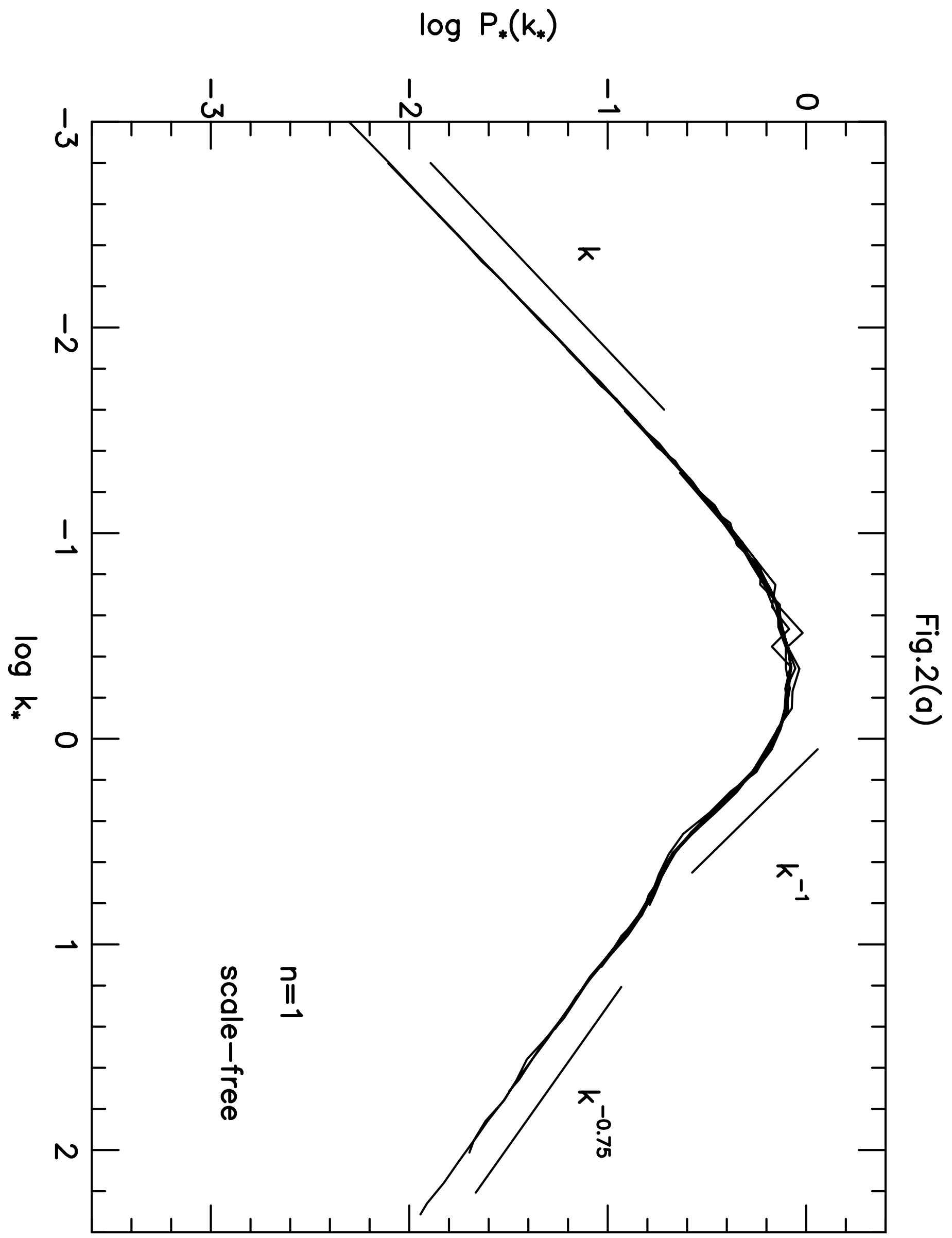




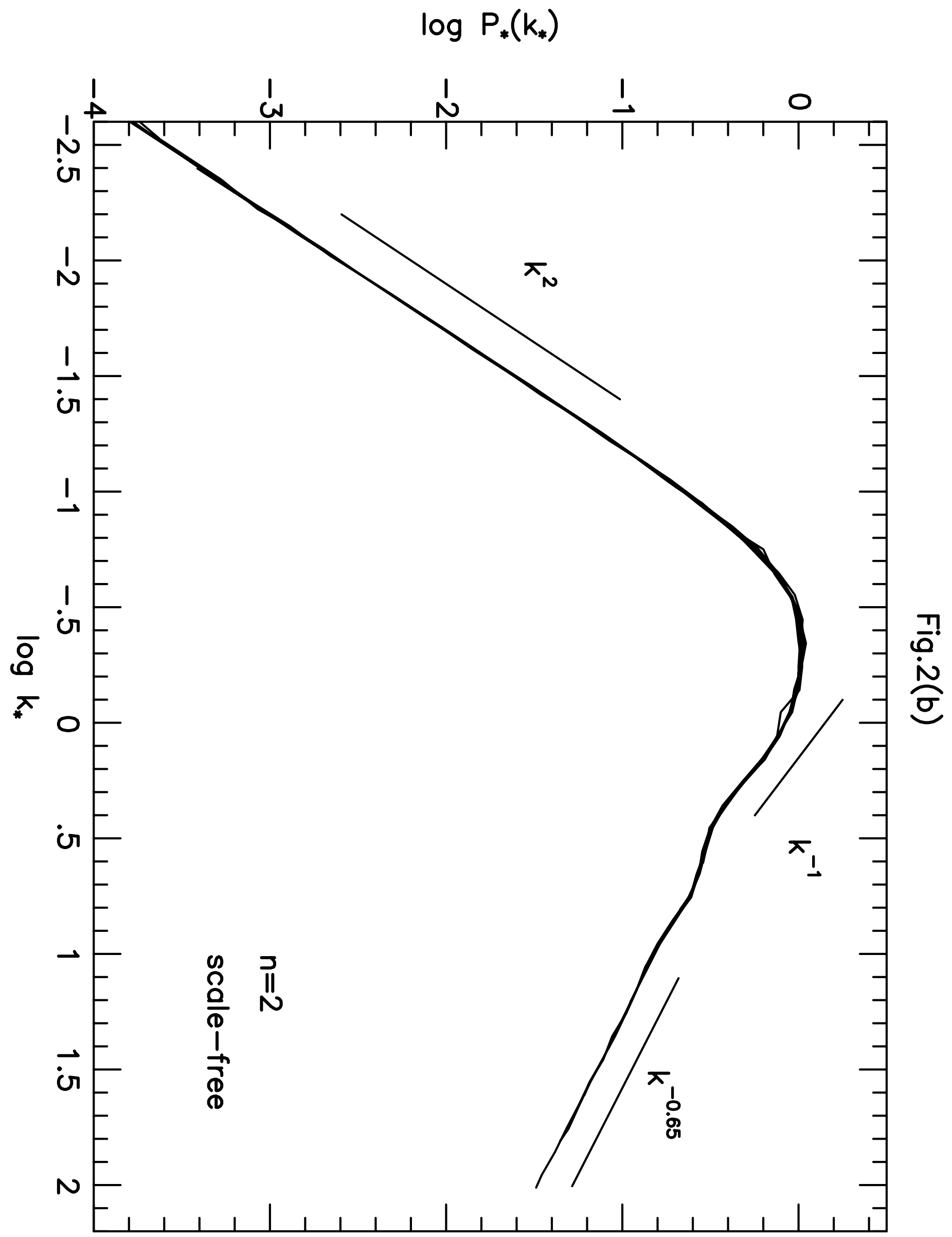




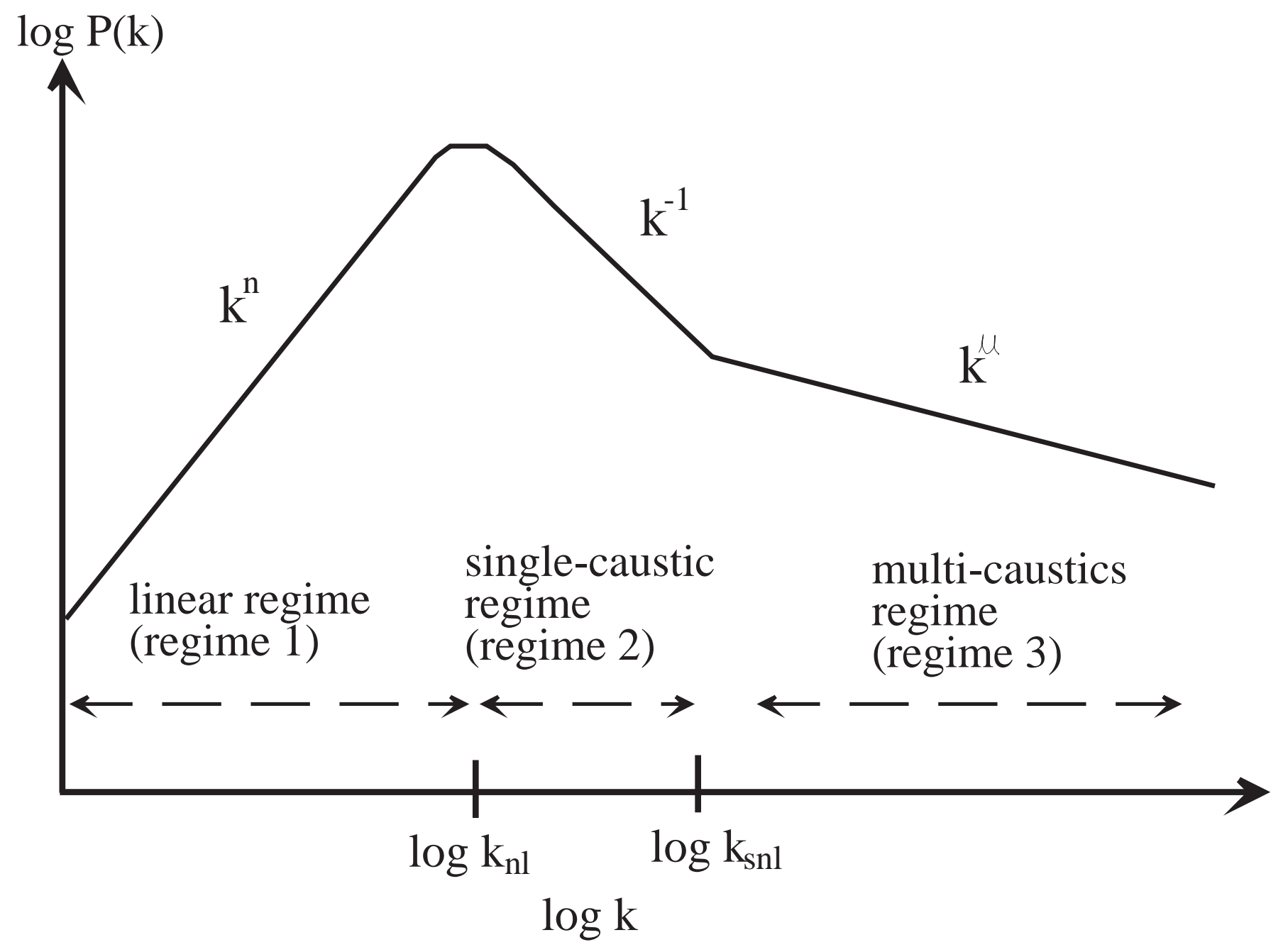

Fig. 3 

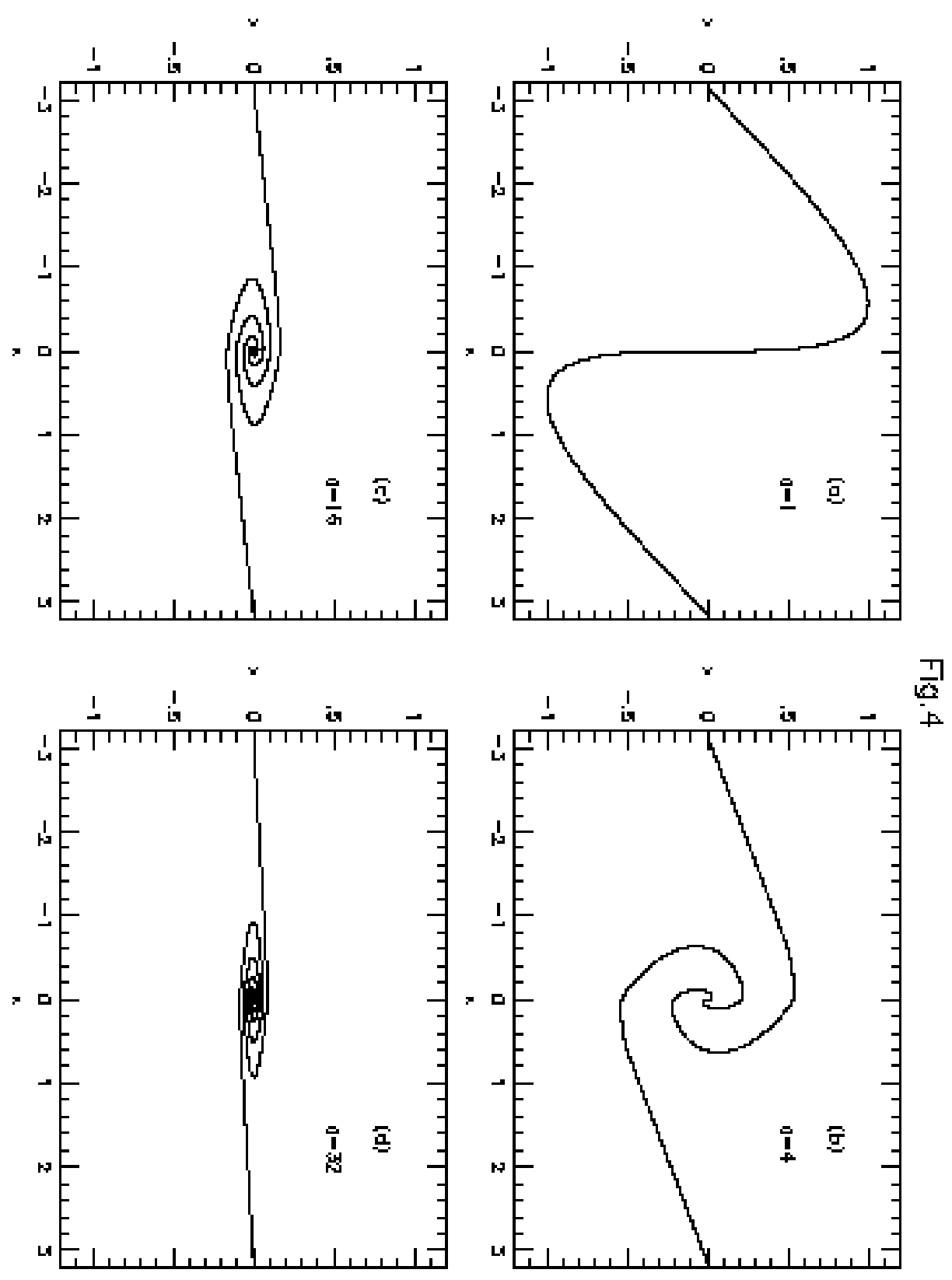


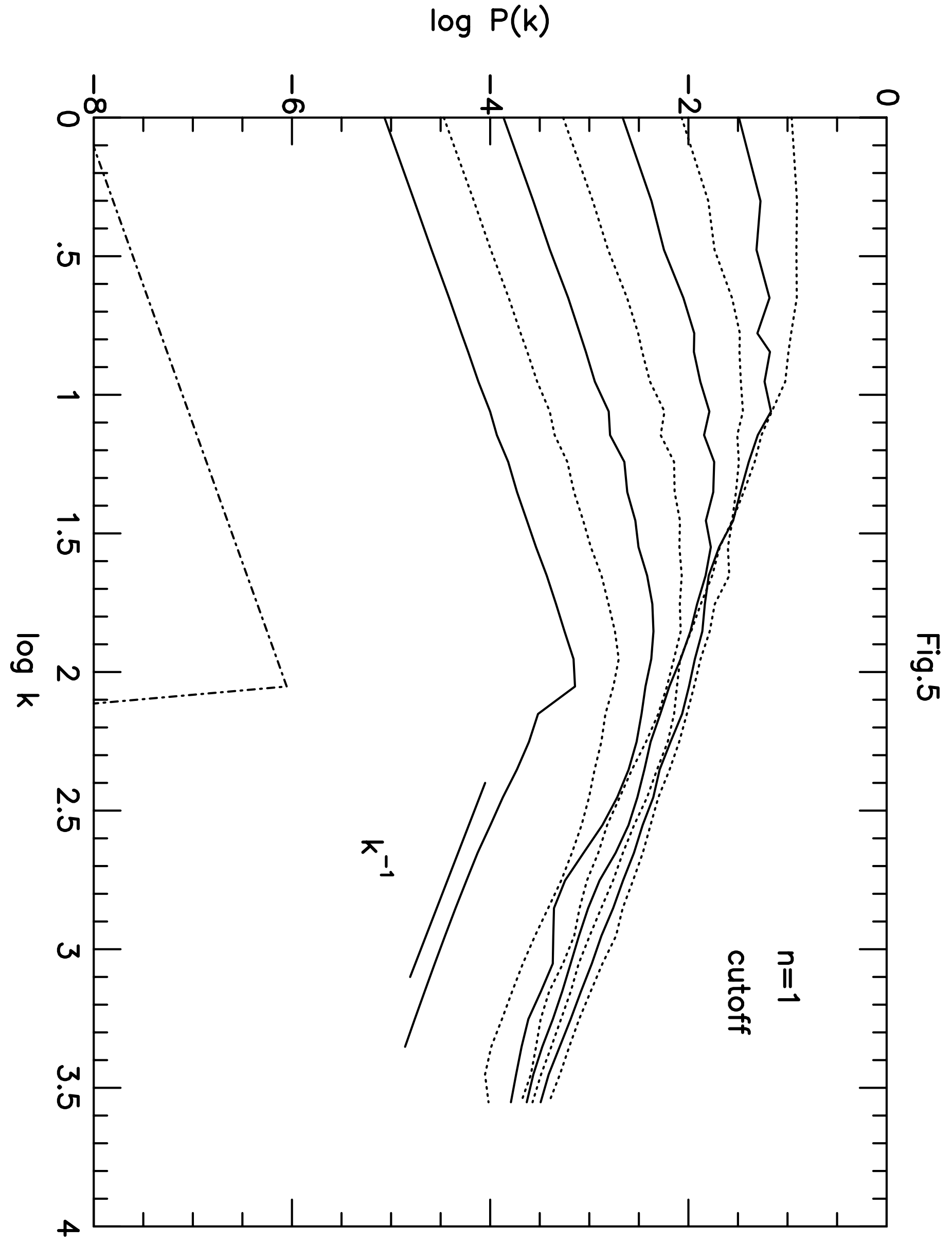




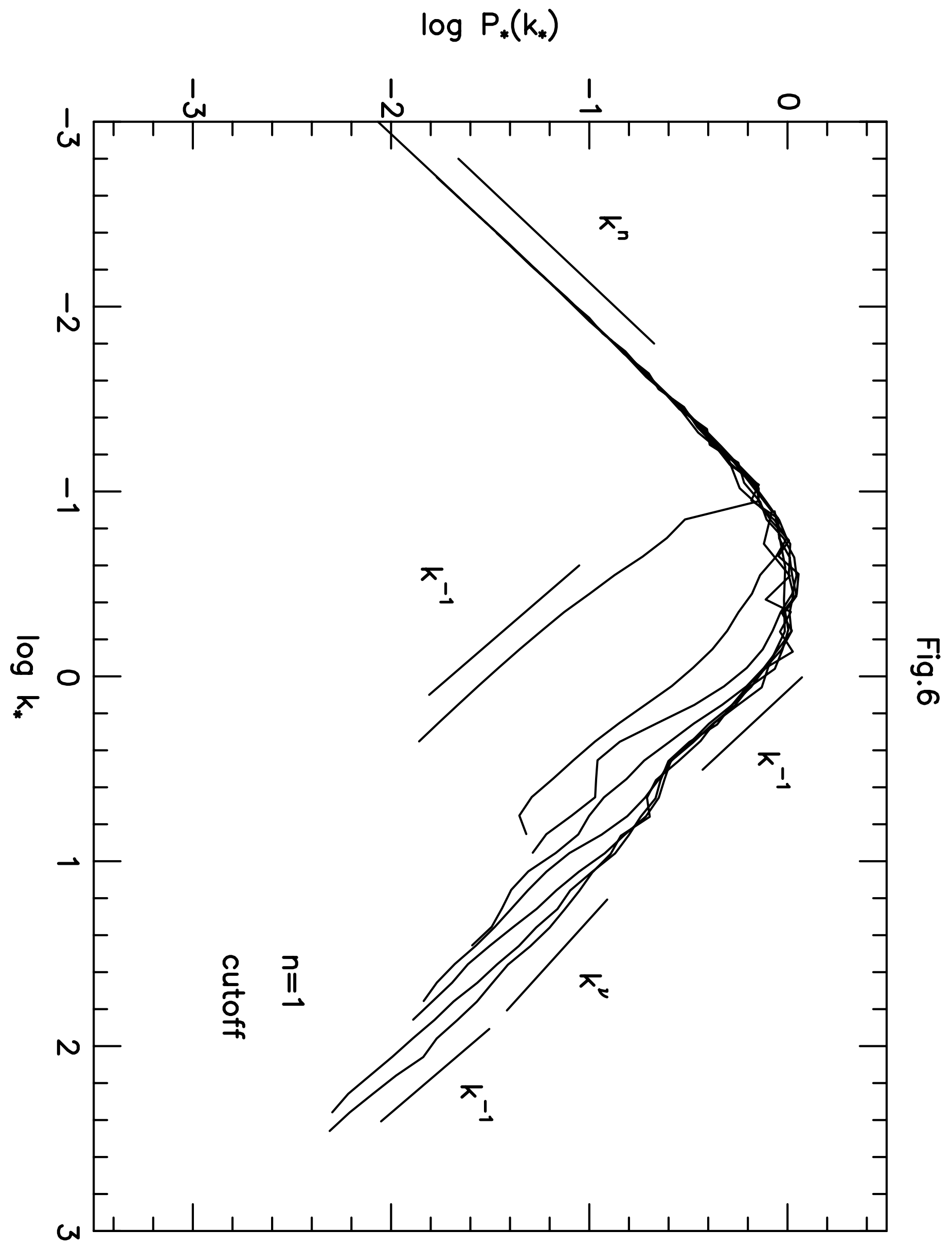




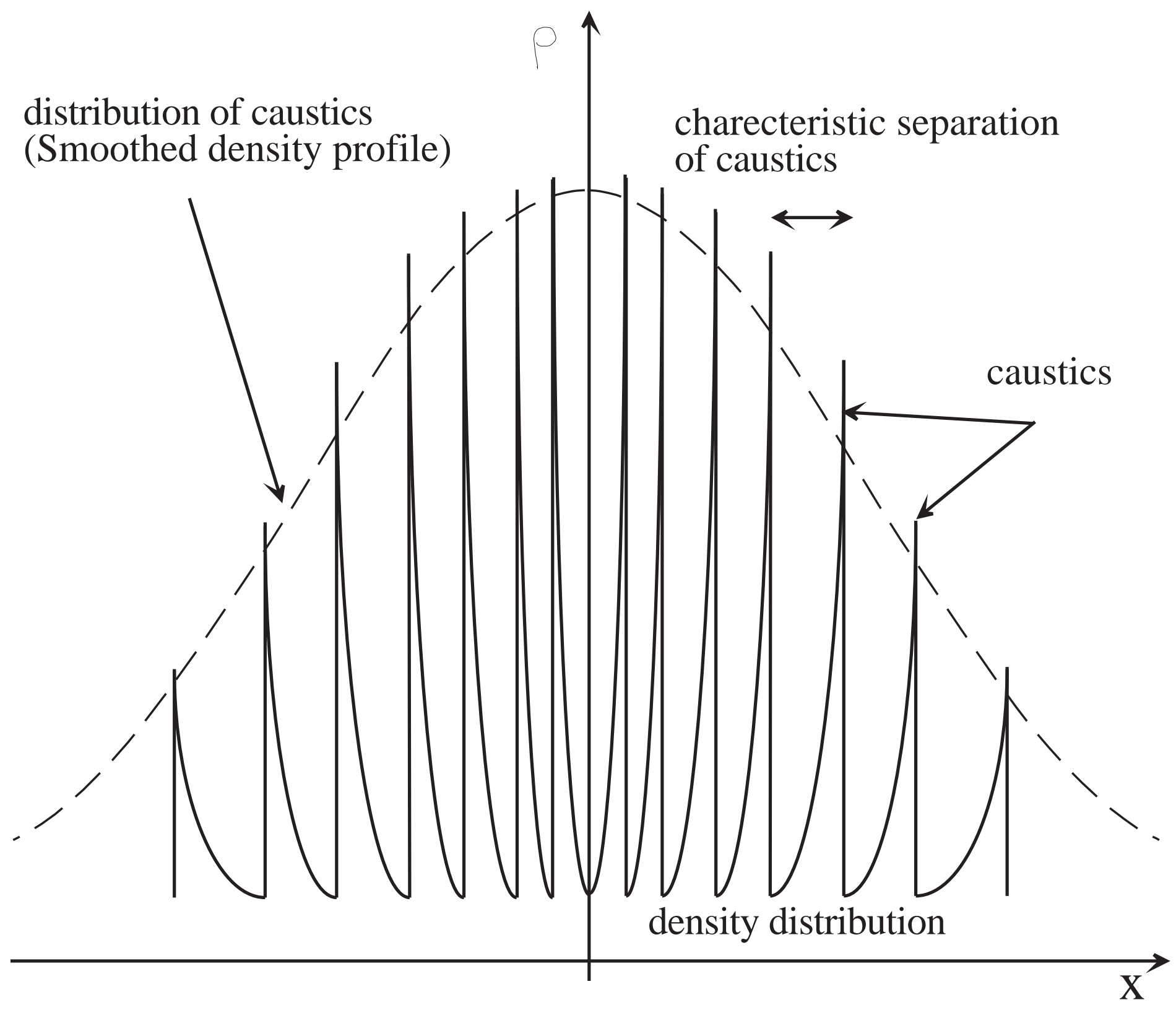

Fig.7 


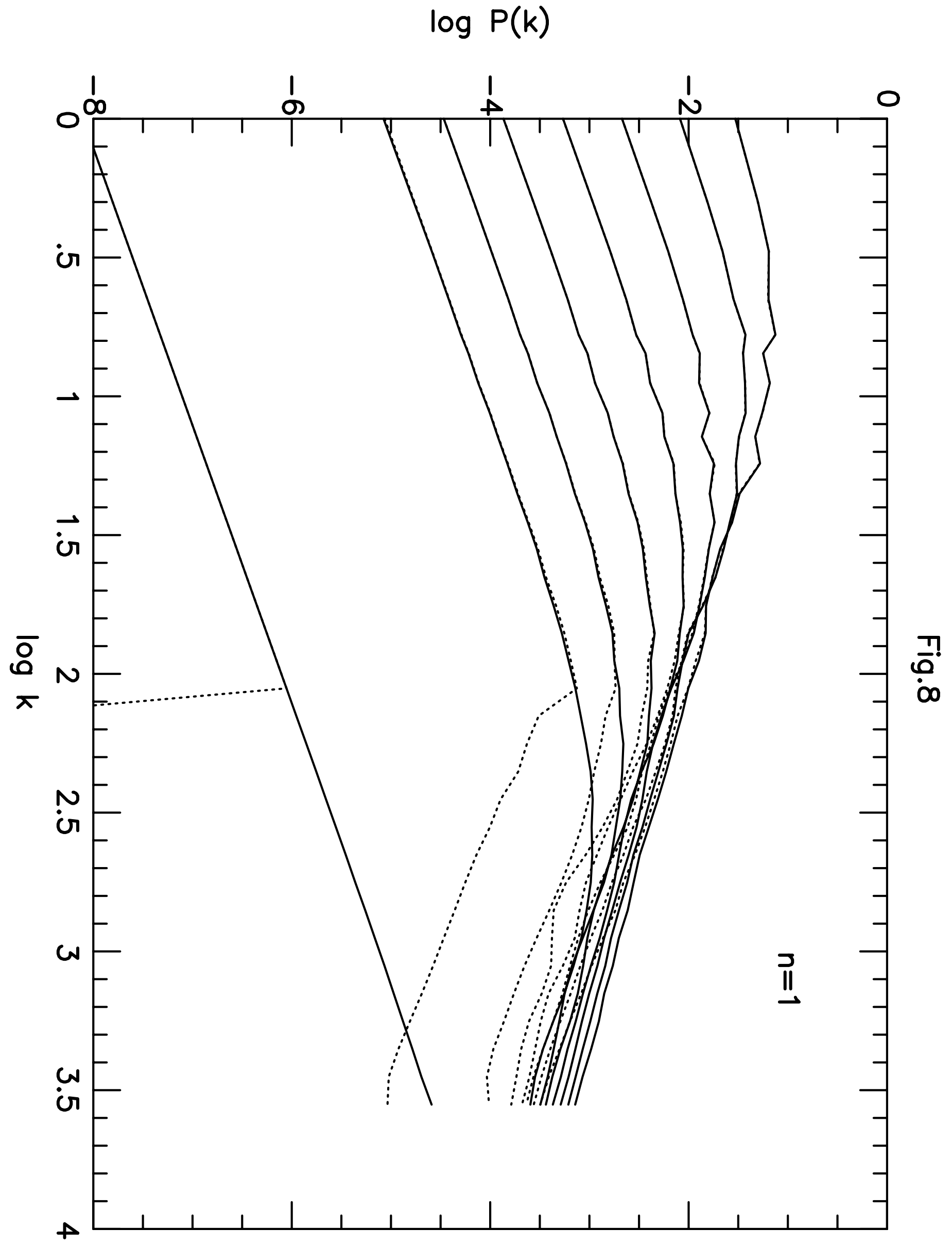




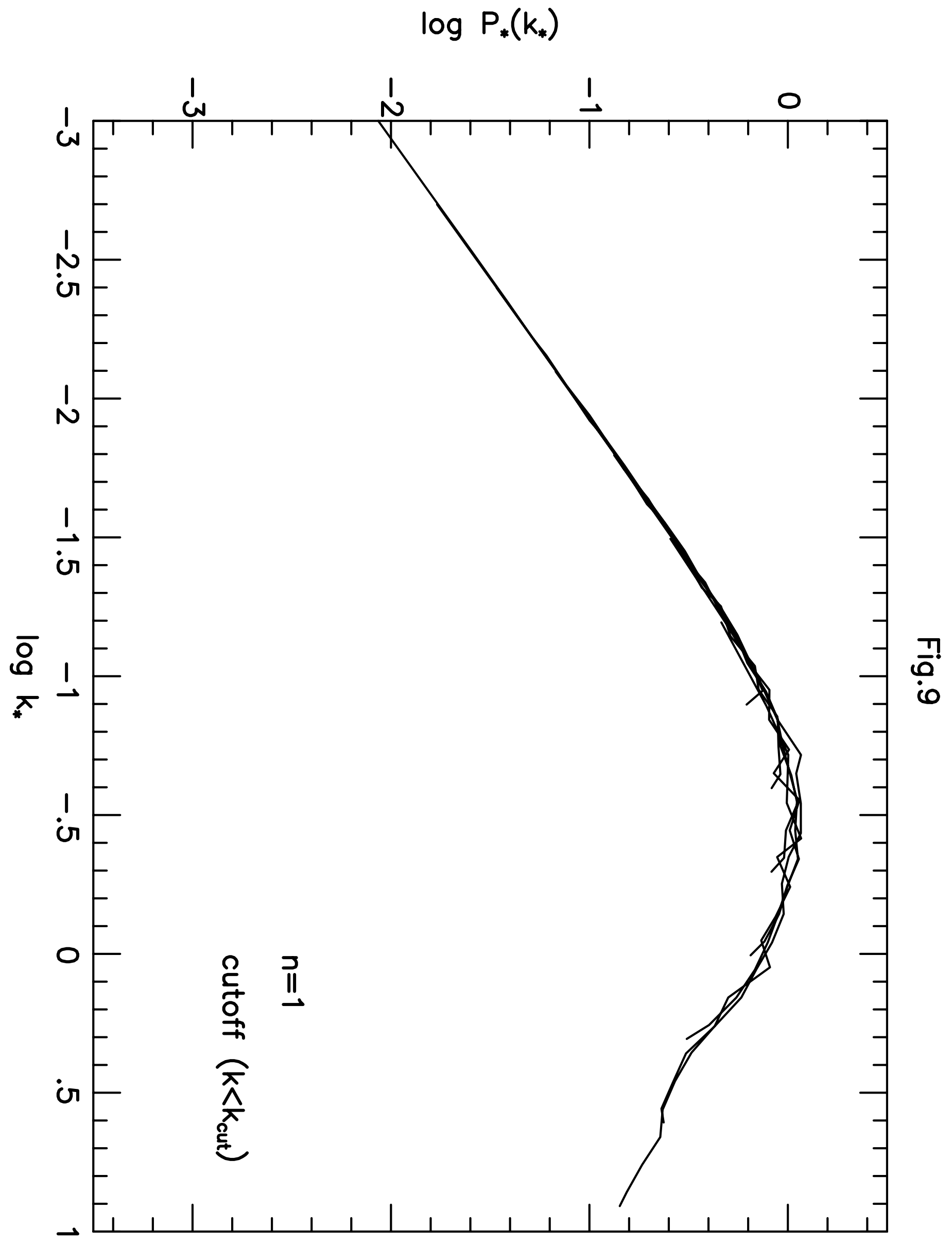




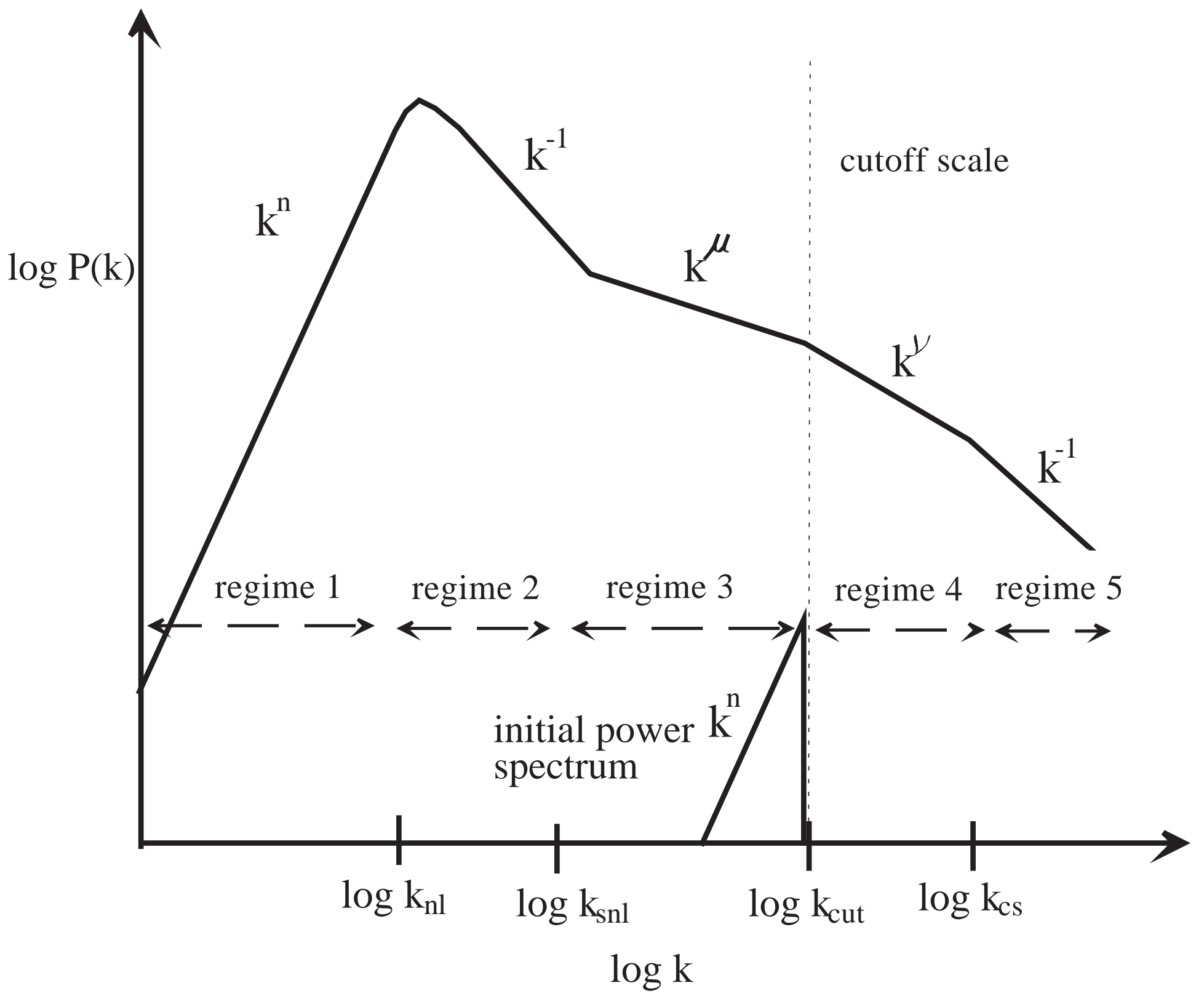

Fig. 10 